\title{
Real-time monitoring of Bacillus subtilis endospore components by attenuated total reflection Fourier-transform infrared spectroscopy during germination
}

\author{
Hon-Yeung Cheung, Jianxun Cui and Su-qin Sunf
}

Research Group for Bioactive Products, Department of Biology \& Chemistry, City University of Hong Kong, Tat Chee Ave, Hong Kong
Author for correspondence: Hon-Yeung Cheung. Tel: +852 2788 7746. Fax: +852 27887406. e-mail : bhhonyun@ cityu.edu.hk

\begin{abstract}
Chemical changes of particular Bacillus subtilis spore components were monitored by attenuated total reflection Fourier-transform infrared spectroscopy (ATR/FTIR) during spore germination on a ZnSe internal reflection element. Within minutes of the initiation of spore germination, significant changes in the amount of calcium dipicolinate (DPA-Ca) and proteins were noted in the wild-type strain. The changes in a germination mutant (strain 1G9, gerD) were similar to those in the wild-type strain, but the rates of change were slower. The changes in another germination mutant (strain 1G7, gerA) were very different from those in the first two strains: germination was slow and incomplete, and proteins and DPA-Ca remained unaltered throughout the course of the germination study. This technique thus offers a sensitive and non-destructive method for real-time monitoring of various cellular components during spore germination.
\end{abstract}

Keywords: Bacillus subtilis, spore, germination mutants, ATR/FTIR

\section{INTRODUCTION}

Germination is a process by which a dormant spore develops into an actively growing vegetative cell. The steps involved in approximate temporal order are: activation, germination initiation, which includes loss of heat resistance, $\mathrm{K}^{+}$and $\mathrm{Zn}^{2+}$ fluxes, release of $\mathrm{Ca}^{2+}$ and dipicolinate (DPA) from the core followed by hydrolysis of cortex peptidoglycan, rehydration of the core protoplast, and resumption of metabolic activity (Dring $\&$ Gould, 1971). Many pleiotropic mutants unable to germinate are defective either in coat structure or in cortex structure. These mutants are blocked at various stages in the germination process (Moir, 1992).

Spore germination has been extensively studied in a number of bacteria, with recent studies of the mechanism focusing on the contribution of biochemical and genetic factors (Keynan, 1978; Jenkinson \& Loard,

†Present address: Analysis Centre, Tsinghua University, Beijing, 100084, China.

Abbreviations: ATR/FTIR, attenuated total reflection Fourier-transform infrared spectroscopy; DPA, dipicolinate; DPA-Ca, calcium dipicolinate.
1983 ; Cheung \& Brown, 1985; Jenkinson, 1988; Preston \& Douthit, 1989; Moir \& Smith, 1990; Johnstone, 1994; Moir et al., 1994; Kuhn et al., 1995; Cheung et al., 1998). Some quantifiable events during germination, such as the loss of heat resistance, the release of DPA and hexosamine-containing cortex fragments, and the change from phase-bright to phase-dark under a phasecontrast microscope, have been described. The loss of heat resistance can be measured by plate counts, and a spectrophotometric assay can be used to measure the release of DPA and $\mathrm{Ca}^{2+}$ (Moir, 1992). However, germination is so rapid that it is not clear whether these events precede other changes associated with initiation of germination. New methods capable of real-time measurement of spore germination are needed.

Attenuated total reflection Fourier-transform infrared spectroscopy (ATR/FTIR) is a sensitive technique providing structural information at the molecular level. Some cellular components of bacteria can be identified by infrared spectroscopy (Helm et al., 1991; Naumann et al., 1991), and constituents such as capsules, DPA and intracellular materials have been detected with this method (Norris \& Greenstreet, 1958; Helm \& Naumann, 1995). Therefore it may offer a method for obtaining useful chemical information during spore 


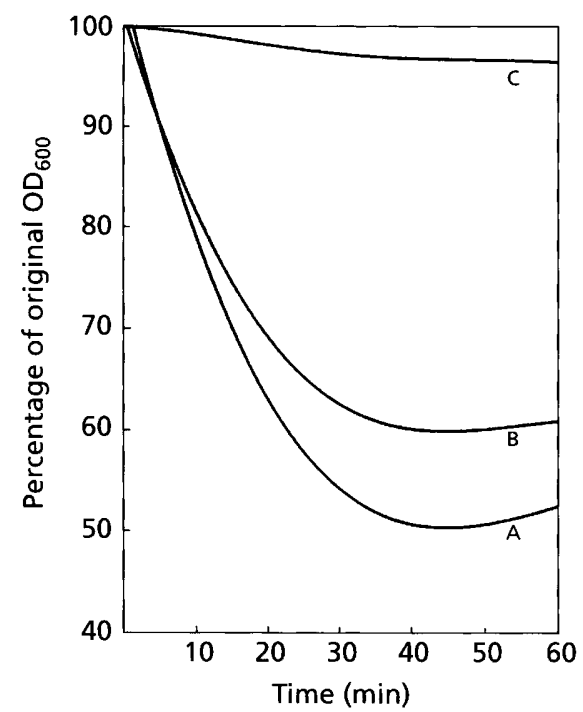

Fig. 1. Germination of spores of $B$. subtilis. Spores of the wildtype strain (curve $A$ ), the gerD mutant (curve $B$ ) and the gerA mutant (curve $C$ ) were induced to germinate in nutrient broth at room temperature as described in Methods. The extent of germination was assessed at $5 \mathrm{~min}$ intervals by measuring the decrease in $\mathrm{OD}_{600}$ of the spore suspension.

germination in real time. The aim of this work was to detect by ATR/FTIR in intact spores the changes in protein, DPA-Ca and polysaccharide that occur during germination of Bacillus subtilis spores.

\section{METHODS}

Bacterial strains. A wild-type strain of Bacillus subtilis was originally obtained from Ernst Freese at the National Institutes of Health, Bethesda, MD, USA; it was designated as 60015 by NIH. Two germination mutants, $1 \mathrm{G} 7$ (gerA11 thr 5 trp C2) and $1 G 9$ (gerD19 trpC2) of B. subtilis were obtained from the Bacillus Genetic Stock Center, Ohio State University, Columbus, USA.

Preparation of spores. Cells of $B$. subtilis were incubated at $37^{\circ} \mathrm{C}$ for $14-16 \mathrm{~d}$ in a modification of the nutrient sporulation medium of Vasantha \& Freese (1979). The medium contained nutrient broth (Oxoid) $8 \mathrm{~g}$ dry base $\mathrm{l}^{-1}, \mathrm{Na}_{2} \mathrm{HPO}_{4} / \mathrm{KH}_{2} \mathrm{PO}_{4}$

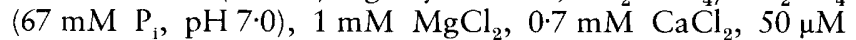
$\mathrm{MnCl}_{2}$ and $1 \mu \mathrm{M} \mathrm{FeCl}_{3}$. Spores were harvested and repeatedly washed with phosphate-buffered saline (PBS) until over $95 \%$ of the cells were free spores. A final spore concentration of approximately $1-5 \times 10^{8}$ spores $\mathrm{ml}^{-1}$ was reconstituted in PBS and kept at $4{ }^{\circ} \mathrm{C}$ until used.

Identification of particular spore constituents by FTIR spectroscopy. A weighed amount of spore pellet spun down from the spores in PBS was added to distilled water to give a final concentration of about $30 \mathrm{mg} \mathrm{ml}^{-1}$. Typically, $20 \mu \mathrm{l}$ of this spore suspension was spread uniformly on a $\mathrm{CaF}_{2}$ crystal plate and dried to a film under moderate vacuum $(2 \cdot 5-7 \cdot 5 \mathrm{kPa})$. The crystal plate was placed on a sample holder in the sample compartment of a FTIR spectrometer (Spectrum 2000, Perkin Elmer), equipped with a DTGS (deuterated triglycine sul-

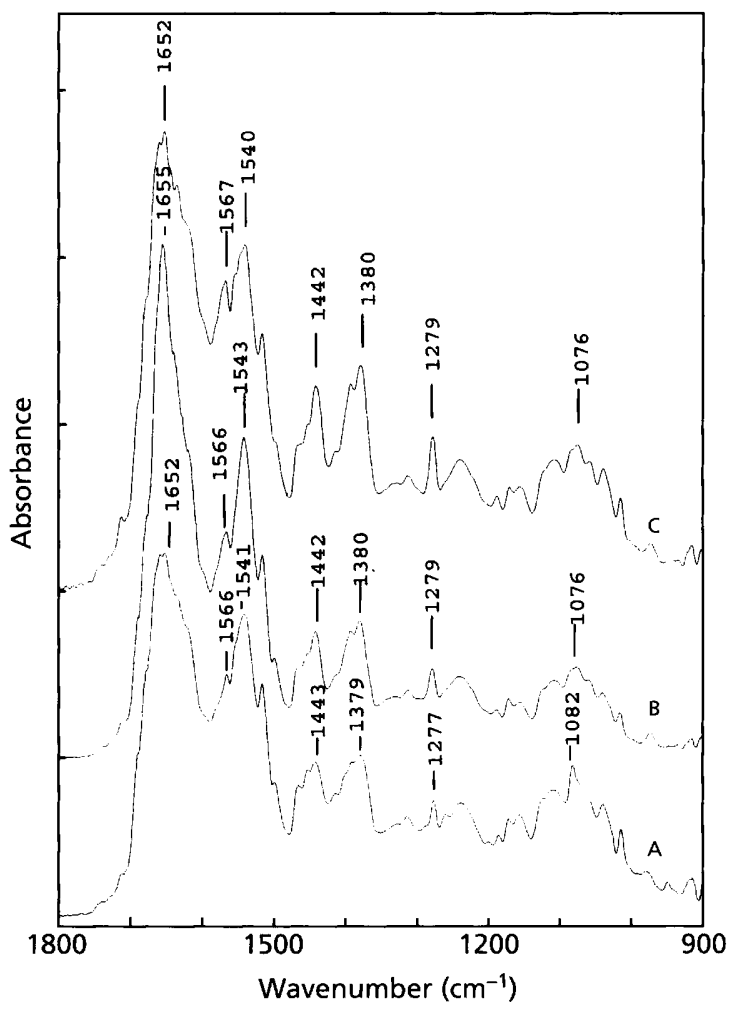

Fig. 2. Deconvoluted FTIR spectra of spores of the three strains of $B$. subtilis. Washed dormant spores were loaded onto a $\mathrm{CaF}_{2}$ crystal plate and freeze-dried under moderate vacuum. Deconvoluted FTIR spectra of the dormant spores of the wildtype strain (curve A); the gerD mutant (curve B) and the gerA mutant (curve C) were derived from the original FTIR spectra obtained directly from the FTIR spectrometer as described in Methods.

phate) detector in the $375-2000 \mathrm{~cm}^{-1}$ range with a nominal resolution of $4 \mathrm{~cm}^{-1}$. Spectra were recorded at room temperature relative to a sample-free crystal, and were the means of 50 scans. The resolution of the spectra was subsequently enhanced by applying a self-deconvolution program, Spectrum version 2.01 (Perkin-Elmer). This software program is analogous to a deconvolution method developed by Kauppinen et al. (1981). Peak positions and spectral resolution were significantly improved by modifying two adjustable parameters, gamma and length, as specified in the software, where gamma is the line-narrowing factor and length is the smoothing applied to limit the noise generated by linenarrowing. Good peak positions of the deconvoluted spectra were observed at gamma value $=4$ and smoothing length $=$ 50 .

Germination study. Germination of the spore suspensions was monitored by measuring the decrease in $\mathrm{OD}_{600}$ (Cheung et al., 1998). Once the spores were mixed with nutrient broth, germination was monitored at $37^{\circ} \mathrm{C}$ and at room temperature $\left(20^{\circ} \mathrm{C}\right)$ by measuring $\mathrm{OD}_{600}$ at $5 \mathrm{~min}$ intervals over a $60 \mathrm{~min}$ period. The rate of germination was confirmed by counting the proportion of phase dark/bright spores. Changes in $\mathrm{OD}_{600}$ were plotted relative to the original $\mathrm{OD}_{600}$ of the control sample. Each data point was the mean of at least two separate experiments. 
(a)
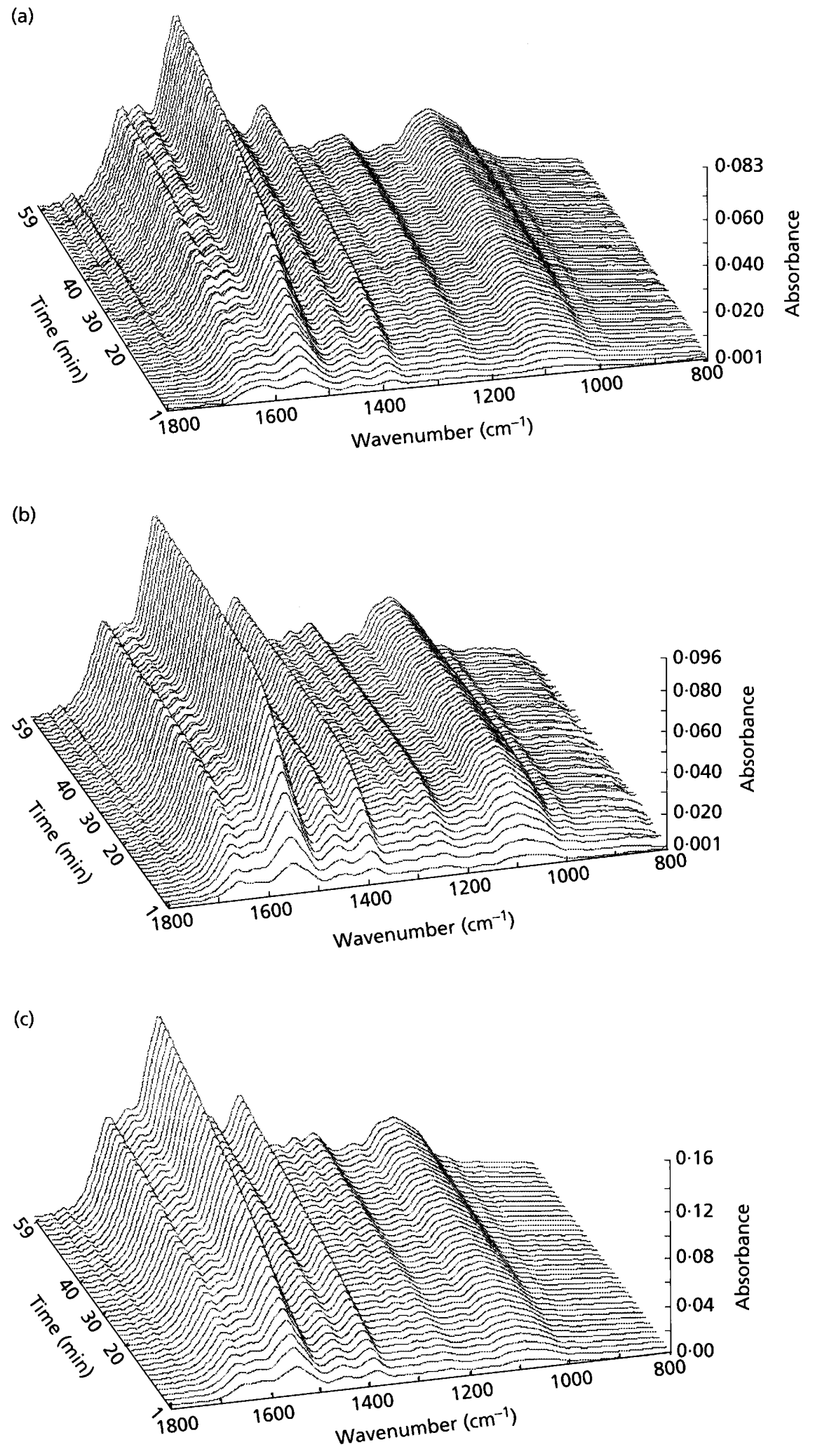

Fig. 3. Dynamic changes in the FTIR spectra of germinating spores. An aliquot of spore suspension of $B$. subtilis in nutrient broth was spread on a ZnSe internal reflection element and the spores were induced to germinate at room temperature as described in Methods. FTIR spectra were taken at 2 min intervals when the spores were germinating. (a) Wild-type strain; (b) gerD mutant; (c) gerA mutant.

Real-time monitoring of chemical components by ATR/FTIR during spore germination. Spores of $B$. subtilis from the stock were suspended in $1 \mathrm{ml}$ nutrient broth. About $0.5 \mathrm{ml}$ of the resuspended spores was immediately put onto the $\mathrm{ZnSe}$ internal reflection element. The element was then placed on the ATR accessory and positioned on an optical bench inside 
the FTIR spectrometer equipped with a $\mathrm{Ge} / \mathrm{KBr}$ beam splitter and DTGS detector. Incubation was carried out at room temperature. The spectrum of one $\mathrm{ZnSe}$ internal reflection element served as a reference, and that of another covered with nutrient broth was used for subtraction from the sample spectra. Spectral resolution was $4 \mathrm{~cm}^{-1}$ and 20 scans were recorded for each spectrum. During germination, spectra were collected at intervals of about $2 \mathrm{~min}$. The spectra were processed by the ATR correction accessory of the Spectrum version 2.01 software. This allows spectra of different types to be compared and yet does not affect qualitative interpretation. It consists of multiplying the absorbance values by a factor $f_{\mathrm{i}}$ that varies with the wavelength $\lambda_{\mathrm{i}}$ and the contact correction factor $C$ according to $f_{\mathrm{i}}=\left(\lambda_{\min }-C\right) /\left(\lambda_{\mathrm{i}}-C\right)$, where $\lambda_{\min }$ is the shortest wavelength in the spectrum. The reason for this is to leave the absorbance value at the shortest wavelength unchanged. As the contact term is increased, bands at longer wavelengths are increasingly attenuated relative to those at shorter wavelengths. Throughout this work, a contact correction factor equal to zero was used.

\section{RESULTS AND DISCUSSION}

\section{Spore germination monitored by optical density}

The germination curves for the three strains of $B$. subtilis in nutrient broth at room temperature $\left(20^{\circ} \mathrm{C}\right)$ are shown in Fig. 1. Decreases in optical density are indicative of the proportion of spores that have germinated (Cheung et al., 1998). As shown by the extent of phase-darkening, spores of the germination mutants were slower to germinate than the wild-type (Moir, 1992). The germination rate of spores of the ger A mutant was significantly slower than that of wild-type spores, while spores of the gerD mutant germinated very slowly.

\section{Identification of particular spore constituents by FTIR spectra}

Consistently reproducible FTIR spectra were obtained with different batches of dormant spores of each of the three strains tested and also with spores of Bacillus stearothermophilus (data not shown). Fig. 2 shows the deconvoluted spectra of spores of the wild-type strain and of the two germination mutants of B. subtilis. The absorption peaks at 1652-1654, 1541-1543 and $1277-1279 \mathrm{~cm}^{-1}$ were assigned to the amide I, II and III bands of proteins respectively (Byler \& Susi, 1986), representing the $\mathrm{C}=\mathrm{O}$ stretching mode and the $\mathrm{N}-\mathrm{H}$ deformation modes of the secondary amide structure. The peak at $1379-1380 \mathrm{~cm}^{-1}$ was due to symmetric stretching vibrations of the carboxyl group of DPA chelated to $\mathrm{Ca}^{2+}$ (Helm \& Naumann, 1995). An additional band at $1566-1570 \mathrm{~cm}^{-1}$ was due to the $\mathrm{C}-\mathrm{N}$ vibrations of the DPA ring. The band near $1443 \mathrm{~cm}^{-1}$ demonstrates the existence of an acid peptide, most probably glutamate and 3-phospho-D-glycerate (Helm $\&$ Naumann, 1995). The absorption peak at $1082 \mathrm{~cm}^{-1}$ was attributed to the ring vibrations of polysaccharide (Cheung et al., 1998).

Although the FTIR spectra for all three strains were very similar, the wild-type and mutants did show some minor differences in the relative intensities and positions of the peaks. The spectra in Fig. 2 imply differences in the relative protein, DPA-Ca and polysaccharide content of the spores of the strains. The ratio of peak heights at wavenumbers 1379 and $1443 \mathrm{~cm}^{-1}$ was higher in spectra of the ger $A$ mutant spores than in those of the other two strains. This indicates that DPA-Ca was more abundant in spores of this mutant than in the other two strains. The band at $1655 \mathrm{~cm}^{-1}$, which is typical of $\alpha$-helical structures, was more abundant in the gerD than in the gerA mutant or the wild-type. The polysaccharide absorbance peaks in the spores of both germination mutants were shifted 6 wavenumbers to lower values than in the wild-type. Since the downward shift of an FTIR peak is an indication of increased co-ordination (Liedberg et al., 1987), this suggests that the molecular structure of a polysaccharide, most probably the peptidoglycan, in the germination mutants was more stable and regular than that in the wild-type.

\section{Dynamic changes in the FTIR spectra of germinating spores}

Structural changes of spore components during germination were monitored for $1 \mathrm{~h}$ in real time by ATR/FTIR. Fig. 3(a-c) shows the three-dimensional maps of FITR spectra of germinating spores of the three $B$. subtilis strains. The increasing absorbance of some functional groups and conformational structures seen in Fig. 3 indicates that their amounts inside the spores increased after exposure to germinants. Although all increased in amount, some did so faster than others. An increase in the intensity of an absorbance peak could have three causes: gradual deposition of spores on the $\mathrm{ZnSe}$ internal reflection element, a structural change in the spores during germination, and probably evaporation of water during the experiment.

The changes of spore components can be observed in Fig. $4(a-c)$, which shows FTIR spectra of germinating spores of $B$. subtilis at the start, and at $15 \mathrm{~min}$ and $1 \mathrm{~h}$ after the start of germination. There was a shift of up to $8 \mathrm{~cm}^{-1}$ for some bands in the spectra of the wild-type strain: from 1443 and $1384 \mathrm{~cm}^{-1}$ at the start to 1451 and $1388 \mathrm{~cm}^{-1}$, respectively, at $1 \mathrm{~h}$. Comparable shifts of up to $7 \mathrm{~cm}^{-1}$ were observed in the gerD mutant: from 1443 and $1384 \mathrm{~cm}^{-1}$ at the start to 1450 and $1388 \mathrm{~cm}^{-1}$, respectively, at $1 \mathrm{~h}$. However, in the spectra of the ger $A$ mutant, no shifts were observed for these peaks. Comparing the relative peak heights at different times of germination with those at the start of the experiment provides information about the rates of change in each component. Within $15 \mathrm{~min}$ of the initiation of spore germination, the amount of protein $\left(1279 \mathrm{~cm}^{-1}\right)$ and chelation state of DPA to $\mathrm{Ca}^{2+}\left(1380 \mathrm{~cm}^{-1}\right)$ were estimated to have changed by $26 \%$ and $11 \%$, respectively, in the wild-type strain. After $1 \mathrm{~h}$ incubation, the amount of protein and the chelation state of DPA had changed by $34 \%$ and $17 \%$, respectively. Changes in the gerD mutant spores were similar to those in the the wild- 


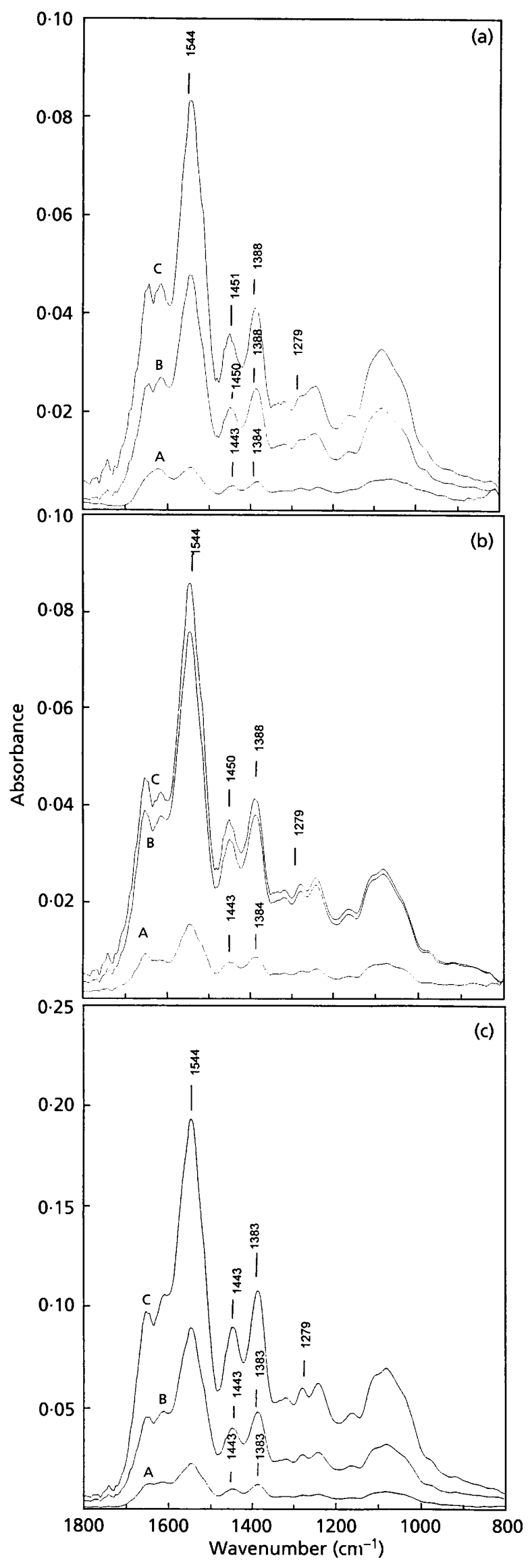

type spores except that they were slower. The chemical changes in the gerA mutant were very different from those in the other two strains. Modification of protein and release of DPA-Ca were not observed in the first $1 \mathrm{~h}$ in this germination mutant. This confirms the gerA mutant's inability to break its dormant state reported previously and matches our optical density measurements.

The above interpretation is supported by the relative intensity changes of the $\mathrm{C}=\mathrm{O}$ anti-symmetric stretching mode relative to that of the neighbouring amide I band near $1650 \mathrm{~cm}^{-1}$ in the FTIR spectra. The intensity of this band, near $1620 \mathrm{~cm}^{-1}$ in Fig. $4(\mathrm{a})$, decreased markedly for the wild-type from the start to $15 \mathrm{~min}$, but it showed no apparent change for the ger $A$ mutant (Fig. 4c) and changed only slightly for the gerD mutant (Fig. 4b).

Degradation of spore protein during germination provides an important source of monomeric substrates for protein synthesis and ribonucleotide metabolism at subsequent stages (Setlow, 1970). The FTIR spectra showed that protein modifications began immediately following the initiation of germination, and approximately $26 \%$ of the spore protein was hydrolysed in the first $15 \mathrm{~min}$ in the wild-type spores. These results are consistent with other authors' observations that about $15-20 \%$ of the total protein in spores was degraded in the first 20 min of germination (Setlow, 1983).

The structural changes in DPA-Ca are normally accompanied by the release of equimolar amounts of $\mathrm{Ca}^{2+}$ and DPA by a germinating spore (Dring \& Gould, 1971). In the past, changes in these molecules have been monitored by differential cathode-ray polarography, which is cumbersome and time-consuming. ATR/FTIR spectroscopy, on the other hand, can provide real-time monitoring of the molecules in a germinating spore. From the intensity of a particular absorbance band in the IR spectra, changes in the functional groups of molecules inside the cells can be followed dynamically in real time and in situ. Thus this method has substantial value for monitoring spore components during germination and for characterizing strains.

\section{ACKNOWLEDGEMENTS}

The authors would like to thank City University of Hong Kong for providing a studentship to Mr Jianxun Cui. They also thank Otto Shek and Sam Li of Perkin Elmer (Hong Kong) Ltd for use of their IR facilities. Their generous help is wholeheartedly acknowledged. The authors are grateful to the editors and the anonymous referees for constructive comments for the improvement of the manuscript.

Fig. 4. FTIR spectra of spores of $B$. subtilis germinating in nutrient broth: at the start (curve A), at 15 min (curve $B$ ) and at $1 \mathrm{~h}$ (curve C). (a) Wild-type strain; (b) gerD mutant; (c) gerA mutant. 


\section{REFERENCES}

Byler, D. M. \& Susi, H. (1986). Examination of the secondary structure of proteins by deconvolved FTIR spectra. Biopolymers 25, 469-487.

Cheung, H. Y. \& Brown, M. R. W. (1985). Coat structure and morphogenesis of bacterial spores in relation to the initiation of spore germination. In Fundamental and Applied Aspects of Bacterial Spores, pp. 227-239. Edited by G. J. Dring, D. J. Ellar \& G. W. Gould. New York: Academic Press.

Cheung, H. Y., So, C. W. \& Sun, S. Q. (1998). Interfering mechanism of sodium bicarbonate on spore germination of Bacillus stearothermophilus. J Appl Microbiol 84, 619-626.

Dring, G. J. \& Gould, G. W. (1971). Sequence of events during rapid germination of spores of Bacillus cereus. J Gen Microbiol 65, 101-104.

Helm, D. \& Naumann, D. (1995). Identification of some bacterial cell components by FTIR spectroscopy. FEMS Microbiol Lett 126, 75-80.

Helm, D., Labischinski, H., Schallehn, G. \& Naumann, D. (1991). Classification and identification of bacteria by Fourier-transform infrared spectroscopy. J Gen Microbiol 137, 69-79.

Jenkinson, H. F. (1988). Altered arrangement of proteins in the spore coat of a germination mutant of Bacillus subtilis. J Gen Microbiol 129, 1945-1958.

Jenkinson, H. F. \& Loard, H. (1983). Protease deficiency and its association with defects in spore coat structure, germination and resistance properties in a mutant of Bacillus subtilis. J Gen Microbiol 129, 2727-2737.

Johnstone, K. (1994). The trigger mechanism of spore germination: current concepts. J Appl Bacteriol Symp Suppl 76, 17S-24S.

Kauppinen, J. K., Moffat, D. S., Mantsch, H. H. \& Cameron, D. (1981). Fourier self-deconvolution: a method for resolving intrinsically overlapped bands. Appl Spectrosc 35, 217-227.

Keynan, A. (1978). Spore structure and its relations to resistance, dormancy and germination. In Spores VII, pp. 43-53. Washington, DC: American Society for Microbiology.
Kuhn, N. J., Setlow, B., Setlow, P., Cammack, R. \& Williams, R. (1995). Cooperative manganese(II) activation of 3-phosphoglycerate mutase of Bacillus megaterium : a biological $\mathrm{pH}$-sensing mechanism in bacterial spore formation and germination. Arch Biochem Biophys 320, 35-42.

Liedberg, B., Carlsson, C. \& Lundstrom, I. (1987). An infrared reflection-absorption study of amino-acids adsorbed on metal surface: L-histidine and L-phenylalanine on gold and copper. $J$ Colloid Interface Sci 120, 113-124.

Moir, A. (1992). Spore germination. In Biology of Bacilli, pp. 23-38. Edited by R. H. Doi \& M. McGloughlin. London: Butterworth-Heinemann.

Moir, A. \& Smith, D. A. (1990). The genetics of bacterial spore germination. Annu Rev Microbiol 44, 531-553.

Moir, A., Kemp, E. H., Robinson, C. \& Corfe, B. M. (1994). The genetic analysis of bacterial spore germination. J Appl Bacteriol Symp Suppl 76, 9S-16S.

Naumann, D., Helm, D. \& Labischinski, H. (1991). Microbiological characterizations by Fourier-Transform infrared spectroscopy (FT-IR). Nature 351, 81-82.

Norris, K. P. \& Greenstreet, J. E. S. (1958). On the infrared absorption spectrum of Bacillus megaterium. J Gen Microbiol 19 , 566-580.

Preston, R. A. \& Douthit, H. A. (1989). Germination of Bacillus cereus: critical control by L-alanine racemase. J Gen Microbiol 130, 3123-3133.

Setlow, P. (1970). Biochemical studies of bacterial sporulation and germination. XXIII. Nucleotide metabolism during spore germination. J Biol Chem 245, 3645-3652.

Setlow, P. (1983). Germination and outgrowth. In The Bacterial Spore II, pp. 211-225. Edited by A. Hurst \& G. W. Gould. New York: Academic Press.

Vasantha, N. \& Freese, E. (1979). The role of manganese in growth and sporulation of Bacillus subtilis. J Gen Microbiol 112, 329-336.

Received 2 April 1998; revised 23 December 1998; accepted 25 January 1999. 